\title{
On the generalized Massey-Rolfsen invariant for link maps
}

\author{
by
}

\author{
A. Skopenkov (Moscow)
}

\begin{abstract}
For $K=K_{1} \sqcup \ldots \sqcup K_{s}$ and a link map $f: K \rightarrow \mathbb{R}^{m}$ let $\widetilde{K}=\bigsqcup_{i<j} K_{i} \times K_{j}$, define a map $\tilde{f}: \widetilde{K} \rightarrow S^{m-1}$ by $\tilde{f}(x, y)=(f x-f y) /|f x-f y|$ and a (generalized) MasseyRolfsen invariant $\alpha(f) \in \pi^{m-1}(\widetilde{K})$ to be the homotopy class of $\tilde{f}$. We prove that for a polyhedron $K$ of dimension $\leq m-2$ under certain (weakened metastable) dimension restrictions, $\alpha$ is an onto or a 1-1 map from the set of link maps $f: K \rightarrow \mathbb{R}^{m}$ up to link concordance to $\pi^{m-1}(\widetilde{K})$. If $K_{1}, \ldots, K_{s}$ are closed highly homologically connected manifolds of dimension $p_{1}, \ldots, p_{s}$ (in particular, homology spheres), then $\pi^{m-1}(\widetilde{K}) \cong$ $\bigoplus_{i<j} \pi_{p_{i}+p_{j}-m+1}^{S}$.
\end{abstract}

1. Introduction. Fix an $s$-tuple $K=\left(K_{1}, \ldots, K_{s}\right)$ of spaces and define $|K|=K_{1} \sqcup \ldots \sqcup K_{s}$. A link map is a map $f:|K| \rightarrow \mathbb{R}^{m}$ such that $f K_{i} \cap f K_{j}=\emptyset$ for each $i \neq j$. This generalization of the usual definition appeared in [Ko 88, Ko 92]. Two link maps $f_{0}, f_{1}:|K| \rightarrow \mathbb{R}^{m}$ are link homotopic if there is a link map $F:|K \times I|=K_{1} \times I \sqcup \ldots \sqcup K_{s} \times I \rightarrow \mathbb{R}^{m} \times I$ such that $F(x, 0)=\left(f_{0}(x), 0\right), F(x, 1)=\left(f_{1}(x), 1\right)$ and $F(x, t) \in \mathbb{R}^{m} \times t$ for each $t$. Two link maps $f_{0}, f_{1}:|K| \rightarrow \mathbb{R}^{m}$ are link concordant if there is a link map $F$ as above with the last condition of level-preserving dropped. In this paper we denote $|K|$ briefly by $K$ (as no confusion can arise).

The problem of classification of link maps up to link concordance and link homotopy was raised in [Mi 54] in an attempt to get a first rough understanding of the overwhelming multitude of classical embedded links up to isotopy. Note that the set of link maps $K \rightarrow \mathbb{R}^{m}$ up to link homotopy depends only on the homotopy type of $K_{1}, \ldots, K_{s}$. An approach to con-

2000 Mathematics Subject Classification: Primary 57Q45, 55S15; Secondary 57M25, 57Q35, 57Q60.

Key words and phrases: deleted product, Massey-Rolfsen invariant, link maps, link homotopy, stable homotopy group, double suspension, codimension two, highly connected manifolds.

Supported in part by the Russian Fundamental Research Foundation Grant No. 9901-00009. 
structing invariants of link homotopy [Sc 68, MR 86, Ko 88] is by analogy to the "deleted product" method in the theory of embeddings (for surveys see $[\operatorname{RS} 96, \S 6, \operatorname{RS} 99, \S 4])$. Let $\widetilde{K}=\bigsqcup_{i<j} K_{i} \times K_{j}$ be the deleted product of the $s$-tuple $K$. For a link map $f: K \rightarrow \mathbb{R}^{m}$ the map $\widetilde{f}: \widetilde{K} \rightarrow S^{m-1}$ is defined by

$$
\widetilde{f}(x, y)=\frac{f x-f y}{|f x-f y|}
$$

Everywhere in this paper we assume that $K$ is homotopy equivalent to a polyhedron and $\operatorname{dim} K \leq m-2 \geq 1$. Then $\operatorname{dim} \widetilde{K} \leq 2(m-2)$, hence the set of maps $\widetilde{K} \rightarrow S^{m-1}$ up to homotopy forms the cohomotopy group $\pi^{m-1}(\widetilde{K}) \cong$ $\bigoplus_{i<j} \pi^{m-1}\left(K_{i} \times K_{j}\right)$. Since $m-1 \geq 2$, it follows that this group does not depend on the choice of base points. This group also depends only on the homotopy type of $K_{1}, \ldots, K_{s}$. For the classical case when $K_{i} \cong S^{p_{i}}$ we have $\left[S^{p_{i}} \times S^{p_{j}}, S^{m-1}\right] \cong \pi_{p_{i}+p_{j}+1-m}^{S}[\mathrm{MR} 86, \S 3]$. Let $\alpha(f)=[\widetilde{f}] \in \pi^{m-1}(\widetilde{K})$ be the generalized Massey-Rolfsen (link homotopy) invariant of $f$.

LEMma 1.0. Let $K=\left(K_{1}, \ldots, K_{s}\right)$ be an s-tuple of polyhedra of dimensions at most $m-2 \geq 1$. If link maps $f_{0}, f_{1}: K \rightarrow \mathbb{R}^{m}$ are link concordant, then $\alpha\left(f_{0}\right)=\alpha\left(f_{1}\right)$ [cf. Ko 88, Proposition 1.10, Ko 92, Theorem C].

Let $\alpha: \operatorname{LM}_{K}^{m} \rightarrow \pi^{m-1}(\widetilde{K})$ be the corresponding map from the set of link concordance classes. For fixed $m$ and $q$ set

$$
\Delta_{r}=2 m-2-2 r-q .
$$

TheOrem 1.1. Let $K=(Q, P, N)$ be a triple of polyhedra of dimensions $q, p$ and $n$ such that $n \leq p \leq q \leq m-2 \geq 1$.

(a) $\alpha: \operatorname{LM}_{K}^{m} \rightarrow \pi^{m-1}(\widetilde{K})$ is surjective if $\Delta_{n} \geq 1$ and either $\Delta_{p} \geq 1$ or $q=2 m-2 p-2 \notin\{2,6,14\}$.

(b) $\alpha: \operatorname{LM}_{K}^{m} \rightarrow \pi^{m-1}(\widetilde{K})$ is bijective if $\Delta_{n} \geq 2$ and $\Delta_{p} \geq 1$.

By the "singular link concordance implies link homotopy" theorem [Me], for $q \leq m-3$ in Theorem 1.1(b) $\mathrm{LM}_{K}^{m}$ can be replaced by the set of link homotopy classes. In the case $s=2$ and $\Delta_{p} \geq 1$, Theorem 1.1(a) was essentially proved in [ST 91, Theorem 3]. Our proof of Theorem 1.1 is based on an extension of the technique from [We 67, ST 91, Sk 97]. Theorem 1.1, its proof and all the remarks below are true for $K=\left(Q, P, N_{1}, \ldots, N_{s}\right)$ where $s=0,1, \ldots$ if the dimension restriction on $n=\operatorname{dim} N$ holds for each $\operatorname{dim} N_{i}$. The extension to more than two components, though not hard, is interesting because in other situations the "triple" invariants can occur for many-component links [Ma 90]. In particular, the dimension restrictions of Theorem 1.1(b) are sharp by [Ma 90, Proposition 8.3]. Theorem 1.1(a) is not true for $q=2 m-2 p-2=6,14$ [Ki 90, Corollary 4.7, cf. SS 92, SSS 98]. For the controlled versions of Theorem 1.1 and the corresponding results on 
embeddings see [ST 91, RS 98]. We conjecture that Theorem 1.1 is true even for compacta $N, P, Q$ (cf. [ST 91, Theorem 3, Sk 98, Theorem 1.4]).

Our proof of Theorem 1.1(a) (resp. (b)) with minor modifications works also for $q=m-1$ and $p \leq(m-2) / 2$ (resp. $p \leq(m-3) / 2)$ [ST 91, Theorem $3, \mathrm{RS}]$. Note that by general position, for this case the set of link maps $K \rightarrow \mathbb{R}^{m}$ up to link homotopy is $\left[Q, \mathbb{R}^{m}-(P \sqcup N)\right]$ (for the only embeddings $\left.P \sqcup N \rightarrow \mathbb{R}^{m}\right)$. It would be interesting to know whether this set is in 1-1 correspondence with $\pi^{m-1}(\widetilde{K}) \cong \pi^{m-1}(Q \times(P \sqcup N))$ : a counterexample would give an example of link maps which are link concordant but not link homotopic (cf. [Sa 99]), while a proof would be an extension of [Ke 59].

For the classical case when $K_{i}$ are spheres Theorem 1.1 is known (but it is interesting that $\mathrm{LM}_{K}^{m}$ is the same for homology spheres $K_{i}$ by Proposition 1.2 (c). Indeed, for codimension $\geq 3$ see [HK 98]. The codimension 2 case for $m$ even is proved simply using general position, the Hilton theorem on homotopy groups of wedges and the James Double Suspension Theorem (cf. [Ki 90, Corollary 4.7]). The codimension 2 case for $m$ odd is reduced, using general position, to the case $s=2$, which is actually proved in [Ko 90, Proposition E] (since $\Sigma \pi_{2 p-1}\left(S^{p}\right) \cong \pi_{p-1}^{S}$; see also [Ne 98]).

Theorem 1.1 together with the following calculations of $\pi^{m-1}(P \times Q)$ (which easily follow from known results) gives some interesting corollaries. In particular, Theorem 1.1 and Proposition 1.2(c) give an analogue of the well-known results on isotopy of highly connected manifolds [We 67, Theorem $4^{\prime}, \mathrm{RS} 96, \S 6$, RS 99, §3]. Denote by $h: \pi^{m-1}(P \times Q) \rightarrow H^{m-1}(P \times Q)$ the cohomology analogue of the Hurewicz homomorphism. We assume $\pi_{l}^{S}=0$ for $l<0$. We omit $\mathbb{Z}$-coefficients from the notation of (co)homology groups. A closed manifold $N$ or a pair $(N, \partial N)$ is called homologically $k$-connected (notation: $N \in \mathrm{HC}_{k}$ or $N \in \partial \mathrm{HC}_{k}$ ) if $H_{i}(N)=0$ for each $i=1, \ldots, k$ or $H_{i}(N, \partial N)=0$ for each $i=0, \ldots, k$, respectively.

Proposition 1.2. Let $P$ and $Q$ be polyhedra of dimensions $p, q \leq m-2$.

(a) If $p+q \leq m-2$, then $\pi^{m-1}(P \times Q)=0$.

(b) If $p+q=m-1$, then $\pi^{m-1}(P \times Q) \cong H^{m-1}(P \times Q) \cong H^{p}(P) \otimes$ $H^{q}(Q)$. If , moreover, both $P$ and $Q$ are connected manifolds, then

$$
\pi^{m-1}(P \times Q) \cong \begin{cases}\mathbb{Z} & \text { if both } P \text { and } Q \text { are closed orientable }, \\ \mathbb{Z}_{2} \quad \text { if both } P \text { and } Q \text { are closed and } \\ & Q \text { is non-orientable, } \\ 0 & \text { if at least one of } P \text { and } Q \text { is non-closed } .\end{cases}
$$

(c) If both $P$ and $Q$ are orientable connected manifolds, then $\pi^{m-1}(P \times Q)$ is isomorphic to 


$$
\begin{cases}\pi_{p+q-m+1}^{S} & \text { if both } P, Q \in \mathrm{HC}_{p+q-m+1} \text { are closed, } \\ H_{p+q-m+1}(Q, \partial Q) & \text { if } P \text { is closed and } Q \in \partial \mathrm{HC}_{p+q-m}, \\ H_{k+1}(P, \partial P) \otimes H_{p+q-m-k}(Q, \partial Q) & \text { if } P \in \partial \mathrm{HC}_{k} \text { and } Q \in \partial \mathrm{HC}_{p+q-m-k-1} .\end{cases}
$$

(d) Suppose that both $P$ and $Q$ are connected manifolds. If one of them is non-closed, then $h$ is an isomorphism for $p+q=m$ and an epimorphism for $p+q=m+1$. If $p+q=m$, then $h$ is an epimorphism whose kernel is either 0 or $\mathbb{Z}_{2}$. If $p+q=m+1$, then the cokernel of $h$ is either 0 or $\mathbb{Z}_{2}$.

(e) The kernel and cokernel of $h$ are always finite.

Proof. (a) follows by general position. (b) and (d) follow by [MT $68, \S 14$ ], since the condition $p+q \geq m$ implies that $m \geq 4$. By [Se 53, Ch. 5, §2, Proposition $\left.2^{\prime}\right], \pi^{m-1}(P \times Q) \cong H^{m-1}(P \times Q)$ modulo the Serre class of finite abelian groups, and (e) follows. Note that [Se 53, Ch. 5, §2, Proposition $\left.2^{\prime}\right]$ is true for even $n$ when $\operatorname{dim} K \leq 2 n$.

In order to prove (c), observe that the obstructions for homotopy of maps $P \times Q \rightarrow S^{m-1}$ are in

$$
\begin{aligned}
H^{l}\left(P \times Q ; \pi_{l}\left(S^{m-1}\right)\right) \cong H_{p+q-l}\left(P \times Q, \partial(P \times Q) ; \pi_{l}\left(S^{m-1}\right)\right) \\
\text { for } l=m-1, m, \ldots, p+q .
\end{aligned}
$$

For the three cases of (c), the only non-trivial group among them is

$$
\left\{\begin{array}{l}
H_{0}\left(P \times Q ; \pi_{p+q}\left(S^{m-1}\right)\right) \cong \pi_{p+q}\left(S^{m-1}\right) \\
H_{p+q-m+1}\left(P \times Q, P \times \partial Q ; \pi_{m-1}\left(S^{m-1}\right)\right) \cong H_{p+q-m+1}(Q, \partial Q) \\
H_{p+q-m+1}\left(P \times Q, \partial(P \times Q) ; \pi_{m-1}\left(S^{m-1}\right)\right) \\
\cong H_{k+1}(P, \partial P) \otimes H_{p+q-m-k}(Q, \partial Q)
\end{array}\right.
$$

The group $\pi^{m-1}(P \times Q)$ can also be calculated using the Postnikov towers, spectral sequences, the Puppe exact sequence for $(P \times Q, P \vee Q)$ (here the formula $T \xi \wedge T \eta \cong T(\xi \times \eta)$ can perhaps be useful) and its dual [MT 68, §14].

In the rest of the introduction we discuss the idea of proof of Theorem 1.1. First we sketch an elementary proof of Theorem 1.1(b) for $m=3$, $p=q=1$ and $N=\emptyset$. From this sketch one can see that here $\mathrm{LM}_{P Q}^{3}$ can be replaced by the set of link homotopy classes. This sketch, though not used in the formal proof, is illustrative because it allows one to visualize in dimension 3 the celebrated 4-dimensional Casson's finger moves.

Sketch of proof that $\alpha: \operatorname{LM}_{P Q}^{3} \rightarrow \pi^{2}(P \times Q) \cong H_{1}(P) \otimes H_{1}(Q)$ is injective for graphs $P$ and $Q$. Since both $H_{1}(P) \otimes H_{1}(Q)$ and the set of link maps $P \sqcup Q$ up to link homotopy depend only on the homotopy type of $P$ and $Q$, we may assume that $P$ and $Q$ are disjoint unions of wedges of circles. So it suffices to prove that the link homotopy class of $f$ depends only on the pairwise linking coefficients of the circles of $P$ and of $Q$. The 
new point with respect to the classical case when both $P$ and $Q$ are circles is that even when $f P \subset \mathbb{R}^{3}$ is unknotted, $\pi_{1}\left(\mathbb{R}^{3}-f P\right)$ is non-commutative and hence the homotopy class of $\left.f\right|_{Q}$ in $\pi_{1}\left(\mathbb{R}^{3}-f P\right)$ is not uniquely defined by those linking coefficients. The example when $P=S^{1} \sqcup S^{1}, Q=S^{1}$ and $f: P \sqcup Q \rightarrow \mathbb{R}^{3}$ is the Borromean rings illustrates this point. It is well known that in this example we can make a homotopy (not an isotopy!) of $\left.f\right|_{P}: P \rightarrow \mathbb{R}^{3}-f Q$ to get a map $f^{\prime}: P \rightarrow \mathbb{R}^{3}-f Q$ so that $f Q$ is unlinked to $f^{\prime} P$, therefore $f$ is link homotopic to a trivial link. In the general case we can make an analogous link homotopy which has the effect of multiplication of the homotopy class of $\left.f\right|_{P}: P \rightarrow \mathbb{R}^{3}-f Q$ or $\left.f\right|_{Q}: Q \rightarrow \mathbb{R}^{3}-f P$ by a commutator. A series of such link homotopies joins our link map $f$ to the standard link map with the same collection of pairwise linking coefficients.

The above link homotopy made $P$ and $Q$ unlinked at the price of selfintersections, just as Casson's finger moves made two proper 2-disks in $D^{4}$ disjoint at the price of self-intersections (cf. the proof of Disjunction Lemma 2.1 for the case $p=q=2, m=4)$. The above link homotopy, completed by the "return" self-intersection of $f^{\prime} P$ far away from $f^{\prime} Q$ and considered as a map $P \times I \rightarrow \mathbb{R}^{3} \times I$, is obtained from the identical homotopy by Casson's finger move.

Formally, Theorem 1.1(a) follows from the case $\partial K=\emptyset$ of Theorem 1.3 below (the general case $\partial K \neq \emptyset$ is used in the proof of Theorem 1.1(b)). In this paper for a polyhedron $K$ we denote by $\partial K$ some subpolyhedron of $K$ (it turns out that when $K$ is a manifold, the subpolyhedron $\partial K$ coincides with the boundary of $K)$. Given subpolyhedra $\partial K_{i} \subset K_{i}$, the $s$-tuple $\partial K=$ $\left(\partial K_{1}, \ldots, \partial K_{s}\right)$ is called a sub-s-tuple of $K$. Set $\partial \widetilde{K}=\bigsqcup_{i<j}\left(\partial K_{i} \times K_{j}\right) \cup$ $\left(K_{i} \times \partial K_{j}\right)$. For a map $f: K \rightarrow B^{m}$ define $\Sigma(f)=\mathrm{Cl}\left\{x \in K:\left|f^{-1} f x\right|>1\right\}$.

TheOREM 1.3. Let $K=(Q, P, N)$ be a triple of polyhedra of dimensions $q, p$ and $n$ such that $n \leq p \leq q \leq m-2 \geq 1, \Delta_{p} \geq 0$ and $\Delta_{n} \geq 1$. Suppose that $\partial K$ is a subtriple of $K$ and $f_{0}: K \rightarrow B^{m}$ a PL map such that $\left.f_{0}\right|_{\partial K}$ is a link map in $\partial B^{m}$ and $f_{0}(K-\partial K) \subset \stackrel{\circ}{B}^{m}$. If there exists a map $\Phi: \widetilde{K} \rightarrow S^{m-1}$ such that $\Phi \simeq \widetilde{f}_{0}$ on $\partial \widetilde{K}$, then there exists a homotopy $f_{t}$ rel $\partial K$ such that $f_{1}$ is a link map, $f_{1}(K-\partial K) \subset \stackrel{B^{m}}{ }$, if either $\Delta_{p} \geq 1$ or $q=2 m-2 p-2 \notin\{2,6,14\}$ then $\widetilde{f}_{1} \simeq \Phi$, and

(Z) for $\Delta_{p} \geq 1$ we have $f_{t}=f_{0}$ on $P$; for $\Delta_{p}=0$, given a polyhedron $Z \subset P$ such that $\Delta_{\operatorname{dim} Z} \geq 1$, we have $\left[\Sigma\left(\left.f_{t}\right|_{P}\right)-\Sigma\left(\left.f_{0}\right|_{P}\right)\right] \cap Z=\emptyset$.

The property $(\mathrm{Z})$ is used not in the applications of Theorem 1.3 but in its proof. The case $N \neq \emptyset$ of Theorem 1.3 follows from the case $N=\emptyset$ by taking $(Q, P)=(P, N)$ and then $(Q, P)=(Q, P \sqcup N)$. Applying Theorem 1.3 for $(Q, P)=(Q, P \sqcup N)$ we take $Z=N$; then by $(\mathrm{Z}),\left.f_{1}\right|_{P \sqcup N}$ will remain a link map and the maps $\widetilde{f}_{1}$ and $\Phi$ will remain homotopic on $P \times N$. This 
is the only place where we need $(\mathrm{Z})$ and the homotopy $f_{t}$ (not only the $\left.\operatorname{map} f_{1}\right)$.

The case $s=2$ of Theorem 1.3 is a generalization of a boundary version of [ST 91, Theorem 3 and $3^{\prime}$, SS 90, Theorem 3] and is also a simplification (i.e. a non-controlled version) of those results. So the proof of the case $s=2$ of Theorem 1.3 is less technical than [ST 91, proof of Theorem 3] and we present it here.

2. Proof of Theorem $\mathbf{1 . 3}$ for $s=2$. We use the notation of [RS 72]. The upper index of a polyhedron indicates its dimension. A map $f: M \rightarrow N$ between manifolds is called proper if $f^{-1} \partial N=\partial M$. First we require two lemmas, which are generalizations of the Whitney trick and, on the other hand, versions of special cases of Theorem 1.3.

Disjunction Lemma 2.1. (a) Suppose that $p \leq q \leq m-2, \Delta_{p} \geq 1$ and $f: D^{p} \sqcup D^{q} \rightarrow D^{m}$ is a PL map such that

(2.1.1) $\left.f\right|_{D^{p}}$ is a proper unknotted embedding into $D^{m}$;

(2.1.2) $f \stackrel{\circ}{D}^{q} \subset \stackrel{\circ}{D}^{m}$ and $f \partial D^{q} \cap f D^{p}=\emptyset$;

(2.1.3) the map $\left.\widetilde{f}\right|_{\partial\left(D^{p} \times D^{q}\right)}$ is null-homotopic.

Then there exists a PL link map $f_{1}: D^{p} \sqcup D^{q} \rightarrow D^{m}$ such that $f_{1}=f$ on $D^{p} \sqcup \partial D^{q}$ and $f_{1} \stackrel{\circ}{D}^{q} \subset \stackrel{\circ}{D}^{m}$.

(b) Suppose that $p \leq q \leq m-2, \Delta_{p}=0, D=D_{1}^{p} \sqcup \ldots \sqcup D_{k}^{p}$, $Q^{\prime}$ is a $q$ polyhedron, $K=\left(D^{p} \sqcup D, D^{q} \cup Q^{\prime}\right)$ and $f:|K| \rightarrow D^{m}$ is a $P L$ map such that $\left.f\right|_{D^{p} \sqcup D}$ is a proper embedding, (2.1.1)-(2.1.3) hold and $f\left(D^{p} \sqcup D\right) \cap f Q^{\prime}=$ $f D \cap f D^{q}=\emptyset$. Then there exists a PL link map $f_{1}:|K| \rightarrow D^{m}$ such that $f_{1}=f$ on $Q^{\prime} \cup \partial\left(D^{q} \sqcup D \sqcup D^{p}\right)$ and $f_{1}\left(\stackrel{\circ}{D}^{p} \sqcup \stackrel{\circ}{D} \sqcup \stackrel{\circ}{D^{q}}\right) \subset \stackrel{\circ}{D^{m}}$.

Realization Lemma 2.2. Suppose that $p, q \leq m-2$, either $\Delta_{p} \geq 1$ or $q=2 m-2 p-2 \notin\{2,6,14\}, f_{0}: D^{p} \sqcup D^{q} \rightarrow D^{m}$ is a PL link map such that (2.1.1) holds and $\Psi: D^{p} \times D^{q} \rightarrow S^{m-1}$ is an extension of $\left.\widetilde{f}_{0}\right|_{\partial\left(D^{p} \times D^{q}\right)}$. Then there exists a homotopy (not link homotopy!) $f_{t}$ rel $D^{p} \sqcup \partial D^{q}$ such that $f_{1}$ is a link map and the homotopy $\widetilde{f}_{t}$ on $\partial\left(D^{p} \times D^{q}\right)$ extends to a homotopy between $\Psi$ and $\widetilde{f}_{1}$ on $D^{p} \times D^{q}$.

Comments on the proof: for $p \leq q \leq m-3$ and $\Delta_{p} \geq 1$, Disjunction Lemma 2.1(a) and Realization Lemma 2.2 were actually proved in [We 67, Proposition 3]; see also [Ha 69, §3, Propositions 1, 2]. In [ST 91] it was shown how to relax the condition $q \leq m-3$ to $q \leq m-2$ in both lemmas. Disjunction Lemma 2.1(b) was proved in [ST 91, Proposition 1.3] (for $q=2$ using the idea of [DRS 91, §5]). Our proof is different in some details and, in the case $p=q=2$ and $m=4$, simpler than in [ST 91]. Note that the part of the proof of Theorem 1.1 that uses this case can be replaced by reference to the elementary sketch in $\S 1$. 
Just as in Realization Lemma 2.2, if in Theorem $1.3, \widetilde{f}_{0}=\Phi$ on $\partial K$, then we can deduce (provided either $\Delta_{p} \geq 1$ or $q=2 m-2 p-2 \notin\{2,6,14\}$ ) not only that $\widetilde{f}_{1} \simeq \Phi$, but also that the homotopy $\widetilde{f}_{t}$ on $\partial \widetilde{K}$ extends to a homotopy between $\Psi$ and $\widetilde{f}_{1}$ on $\widetilde{K}$. The dimension restrictions in Disjunction Lemma 2.1(a) and Realization Lemma 2.2 can be relaxed to " $\Sigma^{\infty}$ : $\pi_{q-1}\left(S^{m-p-1}\right) \rightarrow \pi_{p+q-m}^{S}$ is monomorphic" and " $\Sigma^{\infty}: \pi_{q}\left(S^{m-p-1}\right) \rightarrow$ $\pi_{p+q+1-m}^{S}$ is epimorphic", respectively. When $\left.f\right|_{D^{q}}$ is an embedding, for $\Delta_{p} \geq 1$ and $p \leq q \leq m-3$ we can conclude that $\left.f\right|_{D^{q}}$ is joined to $\left.f_{1}\right|_{D^{q}}$ by an ambient isotopy, but if either $q=m-2$ or $\Delta_{p}=0$, then we cannot (since the dimension assumptions for application of the Penrose-WhiteheadZeeman-Irwin Embedding Theorem are not fulfilled). Note that from the Borromean rings example and its generalization [Ma 90, Proposition 8.3] it follows that in Disjunction Lemma 2.1 we cannot achieve $f_{1}=f$ on $D^{p} \sqcup D$ for $q=2 m-2 p-2 \neq 2,6,14$.

Proof of Disjunction Lemma 2.1(a). By (2.1.1), $D^{m}-f D^{p} \simeq S^{m-p-1}$. The homotopy class $I\left(\left.f\right|_{D^{p}},\left.f\right|_{D^{q}}\right) \in \pi_{q-1}\left(S^{m-p-1}\right)$ of the map $\left.f\right|_{\partial D^{q}}$ : $\partial D^{q} \rightarrow D^{m}-f D^{p}$ is called the coefficient of intersection of $\left.f\right|_{D^{p}}$ and $\left.f\right|_{D^{q}}$. By (2.1.2), the map $\tilde{f}: \partial\left(D^{p} \times D^{q}\right) \rightarrow S^{m-1}$ is well defined. By [We 67, Proposition 1] (the codimension 3 assumption can be weakened to (2.1.1)),

$$
\pm \Sigma^{p} I\left(\left.f\right|_{D^{p}},\left.f\right|_{D^{q}}\right)=[\widetilde{f}] \in \pi_{p+q-1}\left(S^{m-1}\right) .
$$

Then by (2.1.3) we have $\Sigma^{p} I\left(\left.f\right|_{D^{p}},\left.f\right|_{D^{q}}\right)=[\widetilde{f}]=0$. Since $\Delta_{p} \geq 1$, by the Freudenthal Suspension Theorem it follows that $I\left(\left.f\right|_{D^{p}},\left.f\right|_{D^{q}}\right)=0$, i.e. the map $\left.f\right|_{\partial D^{q}}$ extends to a map $f_{1}: D^{q} \rightarrow D^{m}-f D^{p}$.

Proof of Disjunction Lemma 2.1(b). Let $r=m-p-1=q / 2$ and $X=D^{m}-f\left(D^{p} \sqcup D\right)$. The plan of the proof is as follows. First we prove that $\alpha=\left[f: \partial D^{q} \rightarrow X\right] \in \pi_{q-1}(X)$ is a sum of Whitehead products (for $r=1$, a product of commutators). Next we take a collection $\left\{S_{l}^{r}\right\}$ of spheroids generating $\pi_{r}(X)$. Finally, we modify $\left.f\right|_{D^{p} \sqcup D}$ by finger moves to get a proper PL map $f_{1}: D^{p} \sqcup D \rightarrow D^{m}$ such that $f_{1}=f$ on $\partial\left(D \sqcup D^{p}\right)$, the map $f: \partial D^{q} \rightarrow D^{m}-f_{1}\left(D^{p} \sqcup D\right)$ is null-homotopic and $Q^{\prime} \cap f_{1}\left(D^{p} \sqcup D\right)=\emptyset$. Then we take as $\left.f_{1}\right|_{D^{q}}$ any extension of $f: \partial D^{q} \rightarrow D^{m}-f\left(D^{p} \sqcup D\right)$.

Now we realize this plan in detail. Suppose first that $r=1$ (and hence $p=q=2, m=4)$. Since $H_{l}\left(D^{4}\right)=0$ for each $l \geq 1$, it follows from the Mayer-Vietoris sequence that

$$
i \oplus j: H_{1}(X) \cong H_{1}\left(D^{4}-f D\right) \oplus H_{1}\left(D^{4}-f D^{p}\right)
$$

is an isomorphism (here $i$ and $j$ are the inclusion homomorphisms). Since $f D^{q} \cap f D=\emptyset$, it follows that $i(h \alpha)=0$. By (2.1.1), (2.1.3), (I) and the fact that $\Sigma: \pi_{1}\left(S^{1}\right) \rightarrow \pi_{2}\left(S^{2}\right)$ is an isomorphism, we have $I\left(\left.f\right|_{D^{q}},\left.f\right|_{D^{p}}\right)=0$. 
Since also by (2.1.1), the Hurewicz homomorphism $h: \pi_{1}\left(D^{4}-f D^{p}\right) \rightarrow$ $H_{1}\left(D^{4}-f D^{p}\right)$ is an isomorphism, it follows that $j(h \alpha)=0$. Therefore $h \alpha=0$ and by the Hurewicz Theorem, $\alpha$ is a product of commutators.

Now suppose that $r \geq 2$. Take spheres $S^{r}, S_{1}^{r}, \ldots, S_{k}^{r}$ bounding small disks transversal to $f D^{p}, f D_{1}^{p}, \ldots, f D_{k}^{p}$, respectively (by pushing along arcs we may assume that all $S_{l}^{r}$ contain a fixed base point of $\left.X\right)$. Let $S=$ $S_{1}^{r} \vee \ldots \vee S_{k}^{r}$. By the Alexander duality, the inclusion homomorphisms

$$
\begin{gathered}
H_{*}\left(S^{r}\right) \rightarrow H_{*}\left(D^{m}-f D^{p}\right), \quad H_{*}(S) \rightarrow H_{*}\left(D^{m}-f D\right), \\
H_{*}\left(S \vee S^{r}\right) \rightarrow H_{*}(X)
\end{gathered}
$$

are isomorphisms. Since $m-p \geq 3$, it follows that $X, D^{m}-f D$ and $D^{m}-f D^{p}$ are simply connected. Hence

$$
D^{m}-f D^{p} \simeq S^{r}, \quad D^{m}-f D \simeq S, \quad X \simeq S^{r} \vee S
$$

Since $q=2 r$, by the Hilton Theorem on homotopy groups of wedges we have

$$
\pi_{q-1}(X) \cong \pi_{q-1}\left(D^{m}-f D\right) \oplus \pi_{q-1}\left(D^{m}-f D^{p}\right) \oplus W,
$$

where $W$ is generated by Whitehead products. Since $f D^{q} \cap f D=\emptyset$, it follows that the projection of $\alpha$ onto the first summand is zero. The projection of $\alpha$ onto the second summand is $I\left(\left.f\right|_{D^{q}},\left.f\right|_{D^{p}}\right)$. By (2.1.1), (2.1.3), (I) and the hard part of the Freudenthal Suspension Theorem, $I\left(\left.f\right|_{D^{q}},\left.f\right|_{D^{p}}\right)$ is in the subgroup generated by the Whitehead square (for $q=6,14$, is zero). Therefore $\alpha$ is a sum of Whitehead products.

For $r \geq 2$ we have $X \simeq S^{r} \vee S$, so we can take spheroids $S^{r}, S_{1}^{r}, \ldots, S_{k}^{r}$ as generators of $\pi_{r}(X)$. If $r=1$ (or, equivalently, $p=q=2$ and $m=4$ ), we take a triangulation of $D^{p} \sqcup D$ in which $f$ is simplicial. For each 2-simplex $\sigma$ of this triangulation take a circle $S_{\sigma}^{1}$ bounding a small disk transversal to $f \sigma$. By general position we may assume that $S_{\sigma}^{1} \cap S_{\tau}^{1}=\emptyset$ for $\sigma \neq \tau$. For each path $u$ joining the base point of $X$ to a point $x_{\sigma u} \in S_{\sigma}^{1}$ take a loop $S_{\sigma u}^{1}$ obtained from $S_{\sigma}^{1}$ by pushing along the arc $u$. Note that contrary to what was stated in [DRS 91, Proof of Theorem 5.1], the points $x_{\sigma u}$ should depend not only on $\sigma$ but also on $u$; they should be distinct for distinct $u$ to get the required property $u_{i}(0,1] \cap u_{j}(0,1]=\emptyset$. By [DRS 91, Assertion 1 in $\left.\S 5\right]$, the spheres $S_{\sigma u}^{1}$ generate $\pi_{1}(X)$. Since the group $\pi_{1}(X)$ is finitely generated, we can choose from $\left\{S_{\sigma u}^{1}\right\}$ a finite number of generators $S_{l}^{1}$. Note that this construction works also for $r \geq 2$.

Since $\alpha$ is a sum of Whitehead products (for $r=1$, a product of commutators), it follows that $\alpha$ is a sum (for $r=1$, a product) of $\left[S_{l}^{r}, S_{t}^{r}\right]$. So we can take a perforated disk $\delta \subset D^{q}$ and a map $f_{1}: \delta \rightarrow X$ such that $f_{1}=f$ on $\partial D^{q}$ and on every other boundary component of $\delta, f_{1}$ is of the form $w_{l t} \circ v$, where 
(v) $v: S^{q-1}=S^{2 r-1}=S^{r-1} \times B^{r} \cup B^{r} \times S^{r-1} \rightarrow S^{r} \vee S^{r}$ is the map with fibers $S^{r-1} \times S^{r-1}$ and $S^{r-1} \times\{x\}$ and $\{x\} \times S^{r-1}$ for each $x \in \stackrel{B}{ }^{r}$,

(w) $w_{l t}: S^{r} \vee S^{r} \rightarrow S_{l}^{r} \vee S_{t}^{r}$ is a homeomorphism if $l \neq t$ and is the "folding" onto $S_{l}$ if $l=t$.

Suppose that $S_{l}^{r}$ and $S_{t}^{r}$ correspond to two disks $\sigma, \tau$ of $D^{p}, D_{1}^{p}, \ldots, D_{k}^{p}$ (for $r=1$, to two simplices $\sigma, \tau$ of $D^{p} \sqcup D$ ). Take $\operatorname{arcs} a, b \subset D^{m}$ joining interior points of these disks (or simplices) to a point near the base point of $X$. By general position we may assume that these arcs are disjoint (and disjoint for distinct $\sigma, \tau)$ and lie outside $f\left(D^{p} \sqcup D \sqcup Q^{\prime}\right) \cup \delta$ except for their ends. Make finger moves of $\sigma$ and $\tau$ along $a$ and $b$, respectively, for each $\sigma, \tau$. We get a new PL map $f_{1}: D^{p} \sqcup D \rightarrow D^{m}$. Since the $\operatorname{arcs} a, b$ miss $\delta$, it follows that the images of the spheroids $S_{l}^{r}, S_{t}^{r}$ are outside $f_{1}\left(D^{p} \sqcup D\right)$. By general position we may assume that $\operatorname{dim}\left(f_{1} \sigma \cap f_{1} \tau\right) \leq 2 p-m$ and $f_{1} \sigma$ intersects $f_{1} \tau$ transversally. We can represent a regular neighborhood $B^{m}$ of an arbitrary point $c$ of this intersection as the product $B^{2 p-m} \times B^{r+1} \times B^{r+1}$ of balls with $B^{2 p-m} \times 0 \times 0$ corresponding to the intersection, $B^{2 p-m} \times B^{r+1} \times 0$ and $B^{2 p-m} \times 0 \times B^{r+1}$ to $f_{1} \sigma$ and $f_{1} \tau$, respectively. In a neighborhood of $c$ we have the "distinguished" torus $0 \times \partial B^{r+1} \times \partial B^{r+1}$. With appropriate orientations the inclusions of $0 \times \partial B^{l+1} \times y$ and $0 \times y \times \partial B^{l+1}$ into $X_{1}=$ $D^{m}-f_{1}\left(D^{p} \sqcup D\right)$ are homotopic in $X_{1}$ to $S_{l}^{r}$ and $S_{t}^{r}$, respectively. Since the map

$$
w_{i j} \circ v: S^{2 l-1} \rightarrow S^{l} \vee S^{l} \rightarrow\left(0 \times y \times \partial B^{l+1}\right) \vee\left(0 \times \partial B^{l+1} \times y\right)
$$

extends to a map $B^{2 l} \rightarrow 0 \times \partial B^{l+1} \times \partial B^{l+1}$ [Ca 86], it follows that $w_{i j} \circ v$ is null-homotopic in $X_{1}$. So the map $f_{1}: \delta \rightarrow X_{1}$ extends to a map $f_{1}: D^{q} \rightarrow X_{1}$. Evidently, the new map $f_{1}$ is as required.

Proof of Realization Lemma 2.2. Suppose that $f_{0}, f_{1}: D^{p} \sqcup D^{q} \rightarrow D^{m}$ are link maps coinciding on $D^{p} \sqcup \partial D^{q}$. Since $f_{0}=f_{1}$ on $D^{p} \sqcup \partial D^{q}$, it follows that there is a homotopy $f_{t} \operatorname{rel} D^{p} \sqcup \partial D^{q}$. For maps $E, G: D^{p} \times D^{q} \rightarrow S^{m-1}$ and a homotopy $F: \partial\left(D^{p} \times D^{q}\right) \times I \rightarrow S^{m-1}$ such that $F(\cdot, \cdot, 0)=E(\cdot, \cdot)$ and $F(\cdot, \cdot, 1)=G(\cdot, \cdot)$ define the map $H_{E F G}: \partial\left(D^{p} \times D^{q} \times I\right) \rightarrow S^{m-1}$ by

$$
\left.H_{E F G}\right|_{D^{p} \times D^{q} \times 0}=E,\left.\quad H_{E F G}\right|_{D^{p} \times D^{q} \times 1}=G,\left.\quad H_{E F G}\right|_{\partial\left(D^{p} \times D^{q}\right) \times I}=F .
$$

We need to find $f_{t}$ so that $H_{\Phi \widetilde{f}_{t} \widetilde{f}_{1}}$ is null-homotopic. Let

$$
S^{q}=D_{0}^{q} \underset{\partial D_{0}^{q}=\partial D_{1}^{q}}{\cup} D_{1}^{q}
$$

and define a map $h_{f_{0} f_{1}}: S^{q} \rightarrow D^{m}-f D^{p}$ by setting $h_{f_{0} f_{1}}=f_{0}$ on $D_{0}^{q}$ and $h_{f_{0} f_{1}}=f_{1}$ on $D_{1}^{q}$. By (2.1.1), $D^{m}-f D^{p} \simeq S^{m-p-1}$, hence $\left[h_{f_{0} f_{1}}\right] \in$

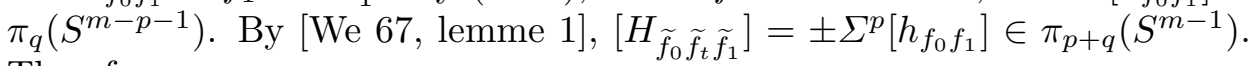
Therefore

$$
\left[H_{\Phi \widetilde{f}_{t} \widetilde{f}_{1}}\right]=\left[H_{\Phi i \widetilde{f}_{0}}\right]+\left[H_{\widetilde{f}_{0} \tilde{f}_{t} \widetilde{f}_{1}}\right]=\left[H_{\Phi i \widetilde{f}_{0}}\right] \pm \Sigma^{p}\left[h_{f_{0} f_{1}}\right] \in \pi_{p+q}\left(S^{m-1}\right) .
$$


Here $\Phi, \widetilde{f}_{0}, \widetilde{f}_{1}$ and $\widetilde{f}_{t}$ denote the restrictions of these maps onto $D^{p} \times D^{q}$ and $\partial\left(D^{p} \times D^{q}\right)$, respectively; $i$ is the constant homotopy. Since for every element $\beta \in \pi_{q}\left(S^{m-p-1}\right)$ there is a map (not necessarily an embedding) $f_{1}: D^{q} \rightarrow D^{m}-f D^{p}$ such that $\left[h_{f_{0} f_{1}}\right]=\beta$, the lemma follows because $\Sigma^{p}: \pi_{q}\left(S^{m-p-1}\right) \rightarrow \pi_{p+q}\left(S^{m-1}\right)$ is an epimorphism. Indeed, the group $\pi_{p+q}\left(S^{m-1}\right)$ is stable. If $\Delta_{p} \geq 1$, then by the Freudenthal Suspension Theorem, $\Sigma^{p}$ is an epimorphism. If $q=2 m-2 p-2 \notin\{2,6,14\}$, then $\Sigma^{2}: \pi_{q}\left(S^{m-p-1}\right) \rightarrow \pi_{q+2}\left(S^{m-p+1}\right)$ is an epimorphism by [Ja 54]. Since $p>1$ (in the opposite case $1 \leq q=2 m-4 \leq m-2$, which is impossible), by the Freudenthal Suspension Theorem, $\Sigma^{p}$ is an epimorphism.

In order to prove Theorem 1.3, take triangulations $T_{P}$ and $T_{Q}$ of $P$ and $Q$ such that $Z$ is a subcomplex of $T_{P}$. The simplices of any triangulation are ordered according to increasing dimension. We use the lexicographic order on the set of pairs of simplices. The case $s=2$ of Theorem 1.3 follows from Proposition 2.3 below for $\sigma^{p}=$ (the last simplex of $T_{P}$ ) and $\sigma^{q}=$ (the last simplex of $T_{Q}$ ). In Proposition 2.3 and its proof the letters $p$ and $q$ denote not $\operatorname{dim} P$ and $\operatorname{dim} Q$ but the dimensions of certain simplices.

Proposition 2.3. Under the assumptions of Theorem 1.3 (where $N=\emptyset$ and $p, q$ are replaced by $\operatorname{dim} P, \operatorname{dim} Q$ ) let $T_{P}, T_{Q}$ be triangulations of $P, Q$, $\sigma^{p} \in T_{P}, \sigma^{q} \in T_{Q}$ any simplices and

$$
J=\partial \widetilde{K} \cup \bigcup\left\{\alpha \times \beta \in T_{P} \times T_{Q} \mid(\alpha, \beta) \leq\left(\sigma^{p}, \sigma^{q}\right)\right\} .
$$

Then there exists a general position $P L$ homotopy $f_{t}$ rel $\partial K$ such that $f_{1}(K-$ $\partial K) \subset \stackrel{\circ}{B}^{m}, f_{1} \alpha \cap f_{1} \beta=\emptyset$ for each $(\alpha, \beta) \subset J,(\mathrm{Z})$ holds and if either $\Delta_{p} \geq 1$ or $q=2 m-2 p-2 \notin\{2,6,14\}$, then $\widetilde{f}_{1} \simeq \Phi$ on $J$.

Proof. By induction on $\left(\sigma^{p}, \sigma^{q}\right)$ we may assume that the conclusion of Proposition 2.3 holds for $f_{1}$ replaced by $f_{0}$ and $J$ replaced by

$$
J_{<}=\partial \widetilde{K} \cup \bigcup\left\{\alpha \times \beta \in T_{P} \times T_{Q} \mid(\alpha, \beta)<\left(\sigma^{p}, \sigma^{q}\right)\right\} .
$$

Suppose that $p+q \geq m-1, \sigma^{p} \not \subset \partial P$ and $\sigma^{q} \not \subset \partial Q$ (otherwise the inductive step holds either by general position or by the inductive hypothesis).

First we show how to achieve $f_{1} \sigma^{p} \cap f_{1} \sigma^{q}=\emptyset$. We begin with the construction of certain balls $D^{m}, D^{p}$ and $D^{q}$, analogous to [We 67, proof of lemme 2, ST 91, proof of Claim on p. 199]. Let $f=f_{0}$. Let $R=\partial P \cup \bigcup\{\alpha \in$ $\left.T_{P} \mid \alpha \leq \sigma^{p}\right\}$. Since $p+q-m+(2 p-m) \leq p$, by general position we have $f^{-1} f \sigma^{q} \cap \Sigma\left(\left.f\right|_{R}\right)=\emptyset$. By general position, $\operatorname{dim}\left(\sigma^{p} \cap f^{-1} f \sigma^{q}\right) \leq p+q-m$. Since $f \alpha \cap f \beta=\emptyset$ for each $\alpha \times \beta \subset J_{<}$, it follows that $f \sigma^{p} \cap f \partial \sigma^{q}=$ $f \partial \sigma^{p} \cap f \sigma^{q}=\emptyset$. Therefore $\sigma^{q} \cap f^{-1} f \sigma^{p} \subset \stackrel{\circ}{\sigma}^{q}$. Let $C_{Q}$ be the trail of $\sigma^{q} \cap f^{-1} f \sigma^{p}$ under a sequence of collapses $\sigma^{q} \searrow\left(\right.$ a point in $\left.\dot{\sigma}^{q}\right)$. Then $C_{Q}$ is collapsible, $C_{Q} \subset \stackrel{\circ}{\sigma}^{q}, \sigma^{q} \cap f^{-1} f \sigma^{p} \subset C_{Q}$ and $\operatorname{dim} C_{Q} \leq p+q-m+1$. 
Analogously we construct a polyhedron $C_{P}$ with the same properties for $q$ and $Q$ replaced by $p$ and $P$.

Since $p+q-m+1+p<m$ and $(p+q-m+1)+(2 p-m)<p$, by general position $C_{P} \cap \Sigma\left(\left.f\right|_{\sigma^{p}}\right)=\emptyset$. This and collapsibility of $C_{P}$ imply collapsibility of $f C_{P}$. Hence the pair $\left(B^{m}, f C_{P}\right)$ is collapsible. Let $C$ be the trail of $f\left(C_{P} \sqcup C_{Q}\right)$ under a sequence of collapses $\left(B^{m}, f C_{P}\right) \searrow 0$. Then the pair $\left(C, f C_{P}\right)$ is collapsible, $C \subset \stackrel{\circ}{B}^{m}, f\left(C_{P} \sqcup C_{Q}\right) \subset C$ and $\operatorname{dim} C \leq p+q-m+2$. Let $D^{m}, D^{q}$ and $D^{p}$ be the regular neighborhoods of $C, C_{Q}$ and $C_{P}$ in some small triangulation of $B^{m}, \sigma^{q}$ and $\sigma^{p}$, respectively. It is easy to verify (2.1.1) (unknottedness of $D^{p}$ follows from [RS 72, Corollary 4.14]), (2.1.2) and

(*) $f \stackrel{\circ}{D}^{q} \subset \stackrel{\circ}{D}^{m}$ and $\sigma^{q} \cap f^{-1} f \sigma^{p} \subset \stackrel{\circ}{D}^{q}$.

Continuation of the proof in the case $\Delta_{p} \geq 1$. Since $(p+q-m+2)+p<m$, by general position we have $\sigma^{p} \cap f^{-1} C=C_{P}$ and $C \cap f\left(R-\stackrel{\circ}{\sigma}^{p}\right)=\emptyset$. Hence

(a) $R \cap f^{-1} D^{m}=\sigma^{p} \cap f^{-1} D^{m}=D^{p}$.

By the PL Annulus Theorem, $\left.\left.\widetilde{f}\right|_{\partial\left(D^{p} \times D^{q}\right)} \simeq \widetilde{f}\right|_{\partial\left(\sigma^{p} \times \sigma^{q}\right)}$ (the meaning of this formally incorrect formula is obvious), and the same for $\widetilde{f} \rightarrow \Phi$.

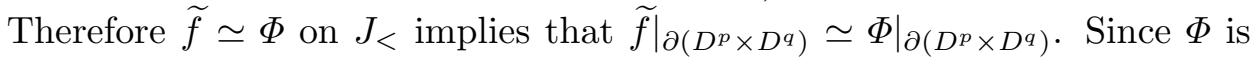
defined over $P \times Q$, we see that $\left.\Phi\right|_{\partial\left(D^{p} \times D^{q}\right)}$ is null-homotopic. This implies (2.1.3). Apply Disjunction Lemma 2.1(a) to get a map $f_{1}: D^{p} \sqcup D^{q} \rightarrow D^{m}$. There exists a homotopy $h_{t}: D^{m} \rightarrow D^{m}$ rel $\partial D^{m}$ such that $h_{1} \circ f=f_{1}$ on $D^{q}$. Define a homotopy $f_{t}: P \sqcup Q \rightarrow B^{m}$ to be $h_{t} \circ f$ on $Q \cap f^{-1} D^{m}$ and $f$ on $P \sqcup\left(Q-f^{-1} D^{m}\right)$. By (a) and since $f_{t}=f$ on $D^{p}$, the conclusion of Proposition 2.3 holds with $J$ replaced by $J_{<}$. From $(*)$ it follows that $f \sigma^{p} \cap f \sigma^{q} \subset \stackrel{\circ}{D}^{m}$. This and (a) imply that $\sigma^{p} \cap f^{-1} f \sigma^{q} \subset \stackrel{\circ}{D}^{p}$. Hence by (*) and Disjunction Lemma 2.1(a), $f_{1} \sigma^{p} \cap f_{1} \sigma^{q}=\emptyset$.

Continuation of the proof in the case $\Delta_{p}=0$. Since $(p+q-m+2)+$ $p=m,(2 p-m)+p<m$ and $2 q-p<p$, by general position we have $R \cap f^{-1} C=C_{P} \cup$ \{points $\left.a_{1}, \ldots, a_{k}\right\}$ and $a_{1}, \ldots, a_{k} \notin T_{P}^{(p-1)} \cup \Sigma\left(\left.f\right|_{R}\right)$. Let $D=D_{1}^{p} \sqcup \ldots \sqcup D_{k}^{p}$ be the regular neighborhood of $\left\{a_{1}, \ldots, a_{k}\right\}$ in some small triangulation of $R$. Hence

(b) $D=D_{1}^{p} \sqcup \ldots \sqcup D_{k}^{p} \subset R,\left.f\right|_{D}$ is an embedding, $R \cap f^{-1} D^{m}=D^{p} \sqcup D$ and $D \cap\left(T_{P}^{(p-1)} \cup \Sigma\left(\left.f\right|_{R}\right) \cup f^{-1} f D^{q}\right)=\emptyset$.

Let $Q^{\prime}=f^{-1} D^{m} \cap\left(\bigcup\left\{\beta \in T_{Q} \mid \beta \leq \sigma^{q}\right\}-\stackrel{\circ}{D}^{q}\right)$. Analogously to the case $\Delta_{p} \geq 1$, (2.1.3) holds. Apply Disjunction Lemma 2.1(b) to get a map $f_{1}$ : $D^{p} \sqcup D \sqcup D^{q} \sqcup Q^{\prime} \rightarrow D^{m}$. There exists a homotopy $f_{t}$ between $\left.f_{0}\right|_{D^{p} \sqcup D \sqcup D^{q} \sqcup Q^{\prime}}$ and $f_{1}$. Since $2 p+q=2 \operatorname{dim} P+\operatorname{dim} Q=2 m-2$, it follows that $p=\operatorname{dim} P$ and $q=\operatorname{dim} Q$, hence neither $\sigma^{q}$ nor $\sigma^{p}$ are contained in the boundary of any simplex of $T_{P}$ or $T_{Q}$. Therefore we can extend the homotopy $f_{t}$ over $P \sqcup Q$ 
by $f$ to obtain a new homotopy $f_{t}: P \sqcup Q \rightarrow B^{m}$. Evidently, the conclusion of Proposition 2.3 holds for $J$ replaced by $J_{<}$(in particular, (Z) follows since $\left.\left(D^{p} \sqcup D\right) \cap T_{P}^{(p-1)}=\emptyset\right)$. From $(*)$, (b) and Disjunction Lemma 2.1(b) it follows that $f_{1} \sigma^{p} \cap f_{1} \sigma^{q}=\emptyset$. For $q \in\{2,6,14\}$ the induction step is proved.

Completion of the proof. Now, assuming that either $\Delta_{p} \geq 1$ or $q=$ $2 m-2 p-2 \notin\{2,6,14\}$, we achieve $\widetilde{f}_{1} \simeq \Phi$ on $J$ and not only on $J_{<}$. Denote by $f$ the map $f_{1}$ obtained above. We begin with the construction of certain balls $D^{m}, D^{p}$ and $D^{q}$. By general position we can take points $c_{P} \in \stackrel{\circ}{\sigma}^{p}-\Sigma(f)$ and $c_{Q} \in \stackrel{\circ}{\sigma}^{q}-\Sigma(f)$ such that the restrictions of $f$ to some small neighborhoods of $c_{P}$ and $c_{Q}$ are locally flat embeddings. Since $\operatorname{dim} P, \operatorname{dim} Q \leq m-2$, we can join points $f c_{P}$ and $f c_{Q}$ by an $\operatorname{arc} c \subset \mathbb{R}^{m}$ such that $c \cap f(P \sqcup Q)=\left\{f c_{P}, f c_{Q}\right\}$. Let $D^{m}$ be a small regular neighborhood of $c$ in $\mathbb{R}^{m}$. Then $f^{-1} D^{m}$ is the disjoint union of PL disks $D^{p} \subset \stackrel{\circ}{\sigma}^{p}$ and $D^{q} \subset$ $\stackrel{\circ}{\sigma}^{q}$, which are regular neighborhoods of $c_{P}$ and $c_{Q}$ in $P \sqcup Q$, respectively. Since the restrictions of $f$ to some small neighborhoods of $c_{P}$ and $c_{Q}$ are locally flat embeddings, we get (2.1.1) and the same for $D^{q}$. By the Borsuk Homotopy Extension Theorem, the map $\Phi$ is homotopic to an extension (denoted also by $\Phi) \Phi: P \times Q \rightarrow S^{m-1}$ of $\left.\widetilde{f}\right|_{J-D^{p} \times D^{q}}$. It follows that $\Phi=\tilde{f}$ on $\partial\left(D^{p} \times D^{q}\right)$. Apply Realization Lemma 2.2 to get the homotopy $f_{t}: D^{p} \sqcup D^{q} \rightarrow D^{m}$. Analogously to the case $\Delta_{p} \geq 1$ above extend $f_{t}$ to $P \sqcup Q$. By the Realization Lemma, $\tilde{f}_{1} \simeq \Phi$ on $J$. Clearly, the conclusion of Proposition 2.3 holds for the composition of the two homotopies constructed. The induction step is proved.

\section{Proofs of Lemma 1.0 and Theorem 1.1(b)}

Cylinder Lemma.

$$
\frac{\widetilde{K \times I}}{\widetilde{K} \times 1 \times 0, \widetilde{K} \times 0 \times 1} \cong \Sigma(\widetilde{K} \times I)
$$

(cf. [Sk, Cylinder Lemma]).

Proof. Represent $\widetilde{K \times I}$ as $\widetilde{K} \times I \times I$. Define a map pr $: \widetilde{K} \times I \times I \rightarrow$ $\Sigma(\widetilde{K} \times I)$ by $\operatorname{pr}(x, y, u, t)=[(x, y,(u+t) / 2), u-t]$. It is easy to see that $\mathrm{pr}$ is a surjection and the only non-trivial preimages of pr are those of the vertices of the suspension and are $\widetilde{K} \times 0 \times 1$ and $\widetilde{K} \times 1 \times 0$. Hence the lemma follows.

Denote by pr: $\widetilde{K \times I} \rightarrow \Sigma(\widetilde{K} \times I)$ the projection of the Cylinder Lemma.

Proof of Lemma 1.0. Let $F: K \times I \rightarrow \mathbb{R}^{m} \times I$ be a link concordance between $f_{0}$ and $f_{1}$. Clearly, $\widetilde{F}(\widetilde{K} \times 0 \times 1), \widetilde{F}(\widetilde{K} \times 1 \times 0)$ and $\widetilde{F}(\widetilde{K} \times\{0 \times 0,1 \times 1\})$ are in the northern and in the southern hemisphere and in the equator $S^{m-1}$ of $S^{m}$, respectively. Therefore by the relative Borsuk Homotopy Extension 
Theorem, $\widetilde{F}$ is homotopic $\operatorname{rel}(\widetilde{K} \times\{0 \times 0,1 \times 1\})$ to a map $\Phi$ such that $\Phi(\widetilde{K} \times$ $0 \times 1)$ and $\Phi(\widetilde{K} \times 1 \times 0)$ are the north and the south pole of $S^{m}$, respectively. Therefore by the Cylinder Lemma there is a map $\Phi^{\prime}: \Sigma(\widetilde{K} \times I) \rightarrow S^{m}$ such that $\Phi=\Phi^{\prime} \circ$ pr. Since $\Phi(\widetilde{K} \times\{0 \times 0,1 \times 1\}) \subset S^{m-1}$, we can modify $\Phi^{\prime}$ by a homotopy $\operatorname{rel}(\widetilde{K} \times\{0 \times 0,1 \times 1\})$ so that $\Phi^{\prime}$ becomes a suspension on $\Sigma(\widetilde{K} \times\{0,1\})$. Since $\operatorname{dim} K \leq m-2$, it follows that $\operatorname{dim}(\widetilde{K} \times I) \leq 2(m-1)-1$. Therefore by the relative Suspension Theorem,

$$
\Sigma: \pi^{m-1}\left(\widetilde{K} \times I, \widetilde{K} \times\{0,1\}, \Phi^{\prime}\right) \rightarrow \pi^{m}\left(\Sigma(\widetilde{K} \times I), \Sigma(\widetilde{K} \times\{0,1\}), \Sigma \Phi^{\prime}\right)
$$

is an epimorphism, i.e. there is a map $\varphi: \widetilde{K} \times I \rightarrow S^{m-1} \operatorname{such}$ that $\varphi=\Phi^{\prime}=$ $\Phi \circ \operatorname{pr}^{-1}=\widetilde{F}$ on $\widetilde{K} \times\{0,1\}$ (and $\Sigma \varphi \simeq \Phi^{\prime}$ on $\Sigma(\widetilde{K} \times I)$ rel $\Sigma(\widetilde{K} \times\{0,1\})$, but we do not need this). So $\varphi$ is the required homotopy between $\widetilde{f}_{0}$ and $\widetilde{f}_{1}$.

Proof of Theorem 1.1(b) (cf. [We 67, §7, Sk 97, §3]). The surjectivity of $\alpha$ follows from Theorem 1.1(a). Suppose that $g_{0}, g_{1}: K \rightarrow \mathbb{R}^{m}$ are link maps such that $\widetilde{g_{0}} \simeq \widetilde{g_{1}}$. Let $G: K \times I \rightarrow I^{m} \times I$ be the linear homotopy between $g_{0}$ and $g_{1}$. Let $\partial(K \times I)=K \times\{0,1\}$. Evidently, $\widetilde{G}$ is defined on $\partial(\widetilde{K \times I})$. Let $\varphi: \widetilde{K} \times I \rightarrow S^{m-1}$ be a homotopy between $\left.\widetilde{G}\right|_{\widetilde{K} \times 0 \times 0}$ and $\left.\widetilde{G}\right|_{\widetilde{K} \times 1 \times 1}$. Define a map $\Phi: \widetilde{K \times I} \rightarrow S^{m}$ by $\Phi=\Sigma \varphi \circ$ pr. Then $\Phi(x, t, y, t)=\varphi(x, y, t)$, hence $\Phi=\widetilde{G}$ on $\widetilde{K} \times\{0 \times 0,1 \times 1\}$. For $(x, t, y, 1) \in K \times[0,1) \times K \times 1$, both $\Phi(x, t, y, 1)$ and $\widetilde{G}(x, t, y, 1)$ are in the northern open hemisphere. For $(x, t, y, 0) \in K \times[0,1) \times K \times 0$, both $\Phi(x, t, y, 0)$ and $\widetilde{G}(x, t, y, 0)$ are in the southern open hemisphere. So for each $(x, s, y, t) \in \partial(\widetilde{K \times I})-(\widetilde{K \times 0} \sqcup \widetilde{K \times 1})$, the points $\Phi(x, s, y, t)$ and $\widetilde{G}(x, s, y, t)$ are not antipodal. Therefore $\Phi \simeq \widetilde{G}$ on $\partial(\widetilde{K \times I})$. Hence we can apply Theorem 1.3 for $K=K \times I, \partial K=K \times\{0,1\}, f_{0}=G$ and $\Phi=\Phi$ (clearly, the dimension restrictions are fulfilled). We obtain a link concordance between $g_{0}$ and $g_{1}$.

Acknowledgments. I would like to thank V. M. Nezhinskiü and S. Melikhov for useful discussions and the referee for his remarks. These results were first presented at the Russian-German Conference dedicated to the 85th birthday of A. D. Alexandrov (St. Petersburg, September 1997) and at the International Conference dedicated to the 60th birthday of S. P. Novikov (Moscow, May 1998).

\section{References}

[Ca 86] A. Casson, Three lectures on new infinite constructions in 4-dimensional manifolds, in: A la Recherche de la Topologie Perdue, L. Guillou and A. Marin (eds.), Progr. Math. 62, Birkhäuser, Boston, 1986, 201-244. 
[DRS 91] A. N. Dranishnikov, D. Repovš and E. V. Shchepin, On intersections of compacta of complementary dimensions in Euclidean space, Topology Appl. 38 (1991), 237-253.

[HK 98] N. Habegger and U. Kaiser, Link homotopy in 2-metastable range, Topology 37 (1998), 75-94.

[Ha 69] L. S. Harris, Intersections and embeddings of polyhedra, ibid. 8 (1969), 1-26.

[Ja 54] I. James, On the iterated suspension, Quart. J. Math. Oxford 5 (1954), 1-10.

[Ke 59] M. Kervaire, An interpretation of $G$. Whitehead's generalization of H. Hopf's invariant, Ann. of Math. 62 (1959), 345-362.

[Ki 90] P. Kirk, Link homotopy with one codimension-two component, Trans. Amer. Math. Soc. 319 (1990), 663-688.

[Ko 88] U. Koschorke, Link maps and the geometry of their invariants, Manuscripta Math. 61 (1988), 383-415.

[Ko 90] —, On link maps and their homotopy classification, Math. Ann. 286 (1990), 753-782.

[Ko 92] —, Homotopy, concordance and bordism of link maps, preprint, Univ. of Siegen, 1992.

[Ma 90] W. Massey, Homotopy classification of 3-component links of codimension greater than 2, Topology Appl. 34 (1990), 269-300

[MR 86] W. Massey and D. Rolfsen, Homotopy classification of higher dimensional links, Indiana Univ. Math. J. 34 (1986), 375-391.

[Me] S. Melikhov, Singular link concordance implies link homotopy in codimension $\geq 3$, submitted.

[Mi 54] J. Milnor, Link groups, Ann. of Math. 59 (1954), 177-195.

[MT 68] R. E. Mosher and M. C. Tangora, Cohomology Operations and Applications in Homotopy Theory, Harper and Row, New York, 1968.

[Ne 98] V. M. Nezhinskiŭ, An analogue of the Milnor group of a link in the theory of multidimensional links, Zap. Nauchn. Sem. POMI 252 (1998), 175-190 (in Russian).

[RS 96] D. Repovš and A. Skopenkov, Embeddability and isotopy of polyhedra in Euclidean spaces, Proc. Steklov Inst. Math. 212 (1996), 163-178.

[RS 98] - - - A deleted product criterion for approximability of maps by embeddings, Topology Appl. 87 (1998), 1-19.

[RS 99] - - , New results on embeddings of polyhedra and manifolds into Euclidean spaces, Uspekhi Mat. Nauk 54 (1999), no. 6, 61-109 (in Russian); English transl.: Russian Math. Surveys, to appear.

[RS] -, -, On projected embeddings and desuspension of the $\alpha$-invariant, submitted.

[RS 72] C. P. Rourke and B. J. Sanderson, Introduction to Piecewise-Linear Topology, Ergeb. Math. Grenzgeb. 69, Springer, Berlin, 1972.

[Sa 99] R. F. Sayakhova, Homotopy classification of singular links of type $(1, \ldots, 1$, $m ; 3)$ with $m>1$, Zap. Nauchn. Sem. POMI 261 (1999), 229-239 (in Russian).

[Sc 68] G. P. Scott, Homotopy links, Abh. Math. Sem. Univ. Hamburg 32 (1968), 186-190.

[SS 90] J. Segal and S. Spież, On transversely trivial maps, Questions Answers Gen. Topology 8 (1990), 91-100.

[SS 92] —, - Quasi-embeddings and embedding of polyhedra in $\mathbb{R}^{m}$, Topology Appl. 45 (1992), 275-282. 
[SSS 98] J. Segal, A. Skopenkov and S. Spież, Embedding of polyhedra in $\mathbb{R}^{m}$ and the deleted product obstruction, Topology Appl. 85 (1998), 335-344.

[Se 53] J. P. Serre, Groupes d'homotopie et classes de groupes abéliens, Ann. of Math. 58 (1953), 258-294.

[Sk 97] A. Skopenkov, On the deleted product criterion for embeddability of manifolds in $\mathbb{R}^{m}$, Comment. Math. Helv. 72 (1997), 543-555.

[Sk 98] -, On the deleted product criterion for embeddability in $\mathbb{R}^{m}$, Proc. Amer. Math. Soc. 126 (1998), 2267-2276.

[Sk] - On the Wu-Haefliger-Hirsch invariants of embeddings and immersions, submitted.

[ST 91] S. Spież and H. Tor uńczy k, Moving compacta in $\mathbb{R}^{m}$ apart, Topology Appl. 41 (1991), 193-204.

[We 67] C. Weber, Plongements de polyèdres dans le domaine metastable, Comment. Math. Helv. 42 (1967), 1-27.

Department of Differential Geometry

Faculty of Mechanics and Mathematics

Moscow State University

Moscow, Russia 119899

E-mail: skopenko@mccme.ru

Received 21 May 1998;

in revised form 2 October 1998 and 14 March 2000 